\title{
A Note on the Extremality of Teichmüller Mappings
}

\author{
By HUANG Xinzhong*) and Hiromi OHTAKE**) \\ (Communicated by Kiyosi ITÔ, M. J. A., April 12, 1995)
}

Introduction. For a hyperbolic Riemann surface $R$, we denote by $A_{2}(R)$ the set of all holomorphic quadratic differentials $\phi=\phi(z) d z^{2}$ on $R$, and set

$$
\begin{aligned}
A_{2}^{p}(R):= & \left\{\phi \in A_{2}(R):\|\phi\|_{p}:=\right. \\
& \left.\left(\int_{R} \lambda_{R}^{2-2 p}|\phi|^{p}\right)^{1 / p}<\infty\right\} \text { for } 1 \leq p<\infty, \\
A_{2}^{\infty}(R):= & \left\{\phi \in A_{2}(R):\|\phi\|_{\infty}:=\right. \\
& \left.\quad \operatorname{ess} \sup _{R} \lambda_{R}^{-2}|\phi|<\infty\right\},
\end{aligned}
$$

where $\lambda_{R}=\lambda_{R}(z)|d z|$ is the hyperbolic metric on $R$ with constant negative curvature -4 . For simplicity, we often write $\|\phi\|_{p, E}$ instead of $\left(\int_{E} \lambda_{R}^{2-2 p}|\phi|^{p}\right)^{1 / p}$.

A quasiconformal mapping $f$ of a Riemann surface $R$ is called extremal if it has the smallest maximal dilatation in the class $Q_{f}$ of all quasiconformal mappings of $R$ which are homotopic to $f$ relative to the border $\partial R$ of $R$. An extremal mapping is called uniquely extremal if there are no other extremal mappings in $\boldsymbol{Q}_{f}$. Hamilton, Reich and Strebel have characterized the extremality: a quasiconformal mapping $f$ is extremal if and only if there is a sequence $\left\{\phi_{n}\right\}_{n=1}^{\infty}$ in $A_{2}^{1}(R),\left\|\phi_{n}\right\|_{1}=$ 1 , such that $\lim _{n \rightarrow \infty} \int_{R} \mu_{f} \phi_{n}=\operatorname{ess} \sup _{R}\left|\mu_{f}\right|$, where $\mu_{f}$ is the Beltrami coefficient of $f$ (Strebel [10]). Such a sequence is called a Hamilton sequence for $f$, and it is said to degenerate if it weakly converges to 0 .

A quasiconformal mapping whose Beltrami coefficient has the form $k \bar{\phi} /|\phi|$, where $0 \leq k$ $<1$ and $\phi \in A_{2}(R) \backslash\{0\}$, is called a Teichmüller mapping corresponding to $\phi$. In the theory of extremal quasiconformal mappings, Teichmüller mappings play an important role. We know that every Teichmüller mapping corresponding to $\phi \in A_{2}^{1}(R)$ is uniquely extremal (Strebel [10]),

*) Department of Applied Mathematics, Huaqiao University, P. R. C.

**) Department of Mathematics, Kyoto University of Education. but there are non-extremal, and extremal but not uniquely extremal Teichmüller mappings (Strebel [8]). So it is expected to find conditions for a holomorphic quadratic differential $\phi$ that guarantees the Teichmüller mapping corresponding to $\phi$ to be extremal or not. For the case $R$ is the unit disk $\boldsymbol{D}$, some extremality theorems have been proved, for instance, Sethares [7], Reich-Strebel [6], Hayman-Reich [2] and one of the authors [3]. On the other hand, Strebel [9] has constructed an example which shows that a lift to the universal covering of an extremal Teichmüller mapping of a compact Riemann surface is not necessarily extremal, and recently McMullen [4] and one of the authors [5] have generalized this.

1. In the present paper, we prove the following:

Theorem 1. Suppose that $R$ is a hyperbolic Riemann surface of finite analytic type, and that $\pi: \tilde{R} \rightarrow R$ is an infinite sheeted regular (i.e. unbounded and unramified) covering from another Riemann surface $\tilde{R}$ to $R$ which satisfies the condition:

(*) for any puncture $a$ of $R$ and any cusped neighborhood $V$ of $a$, there is an integer $m$ such that the restriction of $\pi$ to any connected component of $\pi^{-1}(V)$ is at most $m$ sheeted.

Then for $\Psi \in A_{2}^{\infty}(R), \Psi \neq 0$, and $\psi \in \cup_{1 \leq p<\infty}$ $A_{2}^{p}(\tilde{R})$, the Teichmüller mapping $f_{\pi^{*} \Psi}$ corresponding to the pull-back $\pi^{*} \Psi \in A_{2}^{\infty}(\tilde{R})$ and the Teichmüller mapping $f_{\pi^{*} \Psi+\phi}$ corresponding to $\pi^{*} \Psi+\phi \in$ $A_{2}^{\infty}(\tilde{R})$ have the same Hamilton sequences. In particular, $f_{\pi^{*} \Psi}$ is extremal if and only if so is $f_{\pi^{*} \Psi+\psi}$.

As an application of our Theorem 1 and McMullen's theorem, we have

Corollary 1. Let $\pi: \tilde{R} \rightarrow R$ be a covering as in Theorem 1. If, moreover, $\pi$ is nonamenable, then for any $\Psi \in A_{2}^{\infty}(R) \backslash\{0\}$ and any $\psi \in A_{2}^{p}(\tilde{R})$, $1 \leq p<\infty$, any lifts to the unit disk of the Teichmüller mapping of $\tilde{R}$ corresponding to $\pi^{*} \Psi+\phi$ are not extremal.

Proof. By McMullen's theorem [4], the 
Teichmüller mapping corresponding to $\pi^{*} \Psi$ is not extremal. Thus the Teichmüller mapping corresponding to $\pi^{*} \Psi+\phi$ is not extremal by Theorem 1 , hence its lifts to the unit disk are not extremal.

For a Fuchsian group $\Gamma$ acting on the unit disk $\boldsymbol{D}$, define

$A_{2}^{\infty}(\boldsymbol{D}, \Gamma):=\left\{\phi \in A_{2}^{\infty}(\boldsymbol{D}): \gamma^{*} \phi=\phi\right.$ for all $\left.\gamma \in \Gamma\right\}$.

Corollary 2. If $\Gamma$ is a torsion-free Fuchsian group acting on $\boldsymbol{D}$ such that the Riemann surface $\Gamma \backslash \boldsymbol{D}$ is compact, then for any $\Psi \in A_{2}^{\infty}(\boldsymbol{D}, \Gamma) \backslash\{0\}$ and any $\phi \in A_{2}^{p}(\boldsymbol{D}), 1 \leq p<\infty$, the Teichmiller self-mapping of $\boldsymbol{D}$ corresponding to $\Psi+\psi$ is not extremal.

In particular, there is a non-extremal Teichmüller mapping which is not compatible with any nontrivial Fuchsian groups.

To prove Theorem 1, we need some lemmas. The hyperbolic distance between $a, b \in R$ is denoted by $d_{R}(a, b)$. For $a \in R$ and $l>0$, we set $\Delta(a ; l):=\left\{b \in R: d_{R}(b, a)<l\right\}$. The supremum of all $l>\theta$ for which $\Delta(a ; l)$ is simply connected is called injectivity radius at $a$, and denoted by inj $\operatorname{rad}(a)$.

First of all, by the mean-value theorem for holomorphic functions and Hölder's inequality, we have

Lemma 1. Suppose that $R$ is a hyperbolic Riemann surface and the injectivity radius at $a \in$ $R$ is not less than $l$. Then for all $\phi \in A_{2}(R)$ and $1 \leq p<\infty$,

$$
\left(\lambda_{R}^{-2}|\phi|\right)(a) \leq \frac{1}{\left(\pi \tanh ^{2} l\right)^{1 / p}}\|\phi\|_{p, \Delta(a ; l)} .
$$

Lemma 2. Let $\pi: \tilde{R} \rightarrow R$ be a regular cover. ing of a hyperbolic Riemann surface $R$, and $l_{0}$ be the injectivity radius at $a \in R$. Then for $\phi \in A_{2}^{1}(\tilde{R})$ and $0<l \leq l_{0} / 2$, we have

$\|\phi\|_{1, \pi^{-1}(\Delta(a ; l))} \leq\|\phi\|_{1} \tanh ^{2} l / \tanh ^{2}\left(l_{0} / 2\right)$.

Proof. Let $\tilde{a} \in \pi^{-1}(a)$ and $b \in \Delta(\tilde{a} ; l)$. Since the injectivity radius at $b$ is not less than $l_{0} / 2$, we see $\left(\lambda_{\tilde{R}}^{-2}|\phi|\right)(b) \leq\|\phi\|_{1, \Delta\left(\tilde{a} ; l_{0}\right)} /(\pi$ $\left.\tanh ^{2}\left(l_{0} / 2\right)\right)$ by Lemma 1 . Integrating this on $\Delta(\tilde{a} ; l)$ and summing with respect to $\tilde{a}$, we obtain Lemma 2.

Lemma 3. Let $\pi: \tilde{R} \rightarrow R$ be a regular covering of a hyperbolic Riemann surface $R, a$ be a puncture of $R, V$ be a cusped neighborhood of a which is expressed by $\{0<|z|<1\}$ in terms of a local parameter $z$, and $\cup_{j} \tilde{V}_{j}$ be the decomposition of $\pi^{-1}(V)$ to its connected components. If there is an integer $m$ such that the numbers of sheets of the restrictions $\left.\pi\right|_{\tilde{V} j}$ are bounded by $m$, then we have $\|\phi\|_{\pi^{-1}(\{0<|z|<r\})} \leq C(m)\|\phi\|_{1} r^{1 / m}$ for $\phi \in A_{2}^{1}(\tilde{R})$ and $0<r \leq 1 / 3$, where $C(m)$ is a constant de. pending only on $m$.

Proof. Take a local parameter $\zeta$ on $\tilde{V}_{j}$ in terms of which $\pi(\zeta)=\zeta^{n}$, where $n$ is the number of sheets of the covering $\left.\pi\right|_{\tilde{V} j}: \tilde{V}_{j} \rightarrow V$. Since $\phi=\phi(\zeta) d \zeta^{2}$ has at most a simple pole at $\zeta=0$, by applying the mean-value theorem to $\zeta \phi(\zeta)$, we have $|\phi(\zeta)| \leq C_{0}(m)\|\phi\|_{1, \widetilde{v}_{f}} / \zeta \mid$ for $0<|\zeta|<(1 / 3)^{1 / n}$, from which the assertion follows by the same way as in Lemma 2 .

Proof of Theorem 1. Because $\pi^{*} \Psi, \pi^{*} \Psi+$ $\phi \notin A_{2}^{1}(\tilde{R})$, all Hamilton sequences for $f_{\pi^{*} \Psi}$ and for $f_{\pi^{*} \psi+\psi}$, if any, must degenerate. So it is enough to show that

(1) $\lim _{n \rightarrow \infty} \int_{\tilde{R}}\left|\phi_{n}\right|\left|\frac{\pi^{*} \Psi}{\left|\pi^{*} \Psi\right|}-\frac{\pi^{*} \Psi+\phi}{\left|\pi^{*} \Psi+\phi\right|}\right|=0$

for any sequence $\left\{\phi_{n}\right\}_{n=1}^{\infty} \subset A_{2}^{1}(\tilde{R}),\left\|\phi_{n}\right\|=1$, which is weakly convergent to 0 .

Let $\varepsilon>0$ be a small number. Let $a_{1}, \ldots, a_{k}$ be the punctures of $R$, and $b_{1}, \ldots, b_{l} \in R$ be the zeros of $\Psi$, and take small cusped neighborhoods $V_{1}, \ldots, V_{k}$ of $a_{1}, \ldots, a_{k}$, and small disks $U_{1}, \ldots$, $U_{l}$ centered on $b_{1}, \ldots, b_{l}$ so that they are mutually disjoint. Set $N:=\cup_{j=1}^{k} V_{j} \cup \cup_{j=1}^{l} U_{j}$, and let $\delta$ be the minimum value of $\lambda_{R}^{-2}|\Psi|$ on $R \backslash N$. By Lemmas 2 and 3 , we may assume that $\left\|\phi_{n}\right\|_{1, \pi^{-1}(N)}$ $<\varepsilon$ for any $n$. Take a large compact set $K \subset \tilde{R}$ so that $\lambda_{\tilde{R}}^{-2}|\phi| \leq \varepsilon \delta$ outside $K \cup \pi^{-1}(N)$. By Lemma 1 , we can take such a $K$. Since $|\phi| /$ $\left|\pi^{*} \Psi\right| \leq \varepsilon$ on $\tilde{R} \backslash\left(K \cup \pi^{-1}(N)\right)$, we have

$$
\begin{gathered}
\int_{\tilde{R}}\left|\phi_{n}\right|\left|\frac{\pi^{*} \Psi}{\left|\pi^{*} \Psi\right|}-\frac{\pi^{*} \Psi+\phi}{\left|\pi^{*} \Psi+\phi\right|}\right| \leq \\
\int_{\tilde{R} \backslash\left(K \cup \pi^{-1}(N)\right)} 2 \varepsilon\left|\phi_{n}\right|+\int_{K} 2\left|\phi_{n}\right|+\int_{\pi^{-1}(N)} 2\left|\phi_{n}\right| \\
\leq 2 \varepsilon+2 \int_{K}\left|\phi_{n}\right|+2 \varepsilon .
\end{gathered}
$$

Letting $n \rightarrow \infty$ and $\varepsilon \rightarrow 0$, we obtain (1), and the theorem is proved.

2. To prove (1), the condition (*) is essential. In fact, we can show

Theorem 2. Let $R$ be a (not necessarily analy. tically finite) Riemann surface with a puncture $a, V$ be a cusped neighborhood of $a, \pi: \tilde{R} \rightarrow R$ be a reg. ular covering, and $\left\{\tilde{V}_{j}\right\}_{j}$ be the connected components of $\pi^{-1}(V)$. If the numbers of sheets of the coverings $\left.\pi\right|_{\tilde{V}_{j}}: \tilde{V}_{j} \rightarrow V$ are unbounded, then there 
exist $\phi \in A_{2}^{1}(\tilde{R})$ and a sequence $\left\{\phi_{n}\right\}_{n=1}^{\infty} \subset A_{2}^{1}(\tilde{R})$, $\left\|\phi_{n}\right\|=1$, such that for an arbitrary $\Psi \in A_{2}^{\infty}(R)$, $0<\|\Psi\|_{\infty} \leq 1$,

$$
\begin{aligned}
& \lim _{n \rightarrow \infty} \int_{\tilde{R}} \frac{\overline{\pi^{*} \Psi}}{\left|\pi^{*} \Psi\right|} \phi_{n}=0, \quad b u t \\
& \lim _{n \rightarrow \infty} \int_{\tilde{R}} \frac{\overline{\pi^{*} \Psi}+\bar{\psi}}{\left|\pi^{*} \Psi+\phi\right|} \phi_{n}=1 .
\end{aligned}
$$

Lemma 4. Let $R$ be a Riemann surface, and $a$ $\in R$. If inj $\operatorname{rad}(a) \geq 2 l, l \geq l_{0}:=\log (\sqrt{2}+1)$, then there is $\phi \in A_{2}^{1}(R)$ such that $\|\phi\|_{1}=1$,

$\|\phi\|_{1, R \backslash \Delta(a ; l)} \leq 2^{-1}\left(1-\tanh ^{2} l\right)$, $\lambda_{R}^{-2}|\phi| \geq 2^{-5}\left(1-\tanh ^{2} l\right)^{2}$ on $\Delta(a ; l)$.

Moreover, let $b$ be a point on $R$ for which inj $\operatorname{rad}(b) \geq l_{0}$ and $d_{R}(b, a) \geq l^{\prime}+l_{0}$, then

$$
\left(\lambda_{R}^{-2}|\phi|\right)(b) \leq 1-\tanh ^{2} l^{\prime} .
$$

Proof. Let $\pi: \boldsymbol{D} \rightarrow R$ be a universal covering such that $\pi(0)=a, \Gamma$ be its covering transformation group. Then, by the standard argument and Lemma 1 , it is not difficult to see that $\phi:=$ $\left(\pi^{*}\right)^{-1}\left(\sum_{r \in \Gamma}\left(\gamma^{\prime}\right)^{2} /\left\|\sum_{r \in \Gamma}\left(\gamma^{\prime}\right)^{2}\right\|_{1}\right)$ has the properties in Lemma 4.

Proof of Theorem 2. We may assume that $V=\left\{0<|z|<e^{-2 \pi}\right\}$ and $\lambda_{R}(z)|d z|=(2|z|$ $|\log | z||)^{-1}|d z|$ in terms of a local parameter $z$. Since each $\Psi=\Psi(z) d z^{2}$ has at most a simple pole at $a$, we have $\lambda_{R}^{-2}(z)|\Psi(z)| \leq C_{1}|z|$ $\left.|\log | z\right|^{2}$, where $C_{1}$ is a universal constant.

Let $\left\{l_{n}\right\}_{n=1}^{\infty}$ be a sequence such that $l_{n} \geq l_{0}$ and $\lim l_{n}=\infty$, and define a sequence of large numbers $\left\{l_{n}^{\prime}\right\}_{n=1}^{\infty}$ so that $1-\tanh ^{2} l_{n}^{\prime} \leq 2^{-(2 n+7)}$ $\left(1-\tanh ^{2} l_{n}\right)^{2}$. Our assumption on the numbers of sheets of the coverings implies that we can take disks $\Delta_{n}^{\prime}:=\Delta\left(a_{n} ; l_{n}+l_{n}^{\prime}\right)$ in $\pi^{-1}\left(\left\{C_{1}|z|\right.\right.$ $\left.\left.|\log | z||^{2} \leq 2^{-(2 n+7)}\left(1-\tanh ^{2} l_{n}\right)^{2}\right\}\right)$. We may assume that these disks $\left\{\Delta_{n}^{\prime}\right\}_{n=1}^{\infty}$ are mutually disjoint. Let $\phi_{n} \in A_{2}^{1}(\tilde{R})$ be the holomorphic quadratic differentials obtained by applying Lemma 4 , and set $\phi:=\sum_{n=1}^{\infty} 2^{-n} \phi_{n} \in A_{2}^{1}(\tilde{R})$. Since $\lambda_{\tilde{R}}^{2}$ $\left|\pi^{*} \Psi\right| \leq 2^{-(2 n+2)} \lambda_{R}^{-2}\left|\phi_{n}\right|$ and $\lambda_{\tilde{R}}^{-2}\left|\sum_{k \neq n} 2^{-k} \phi_{k}\right|$ $\leq 2^{-(2 n+2)} \lambda_{R}^{-2}\left|\phi_{n}\right|$ on $\Delta_{n}:=\Delta\left(a_{n} ; l_{n}\right)$, we have $\left|\left(\pi^{*} \Psi+\psi\right) /\right| \pi^{*} \Psi+\phi\left|-\phi_{n} /\right| \phi_{n}|| \leq 2^{-n}$.

Thus we see

$$
\begin{aligned}
& \left|1-\int_{\tilde{R}} \frac{\overline{\pi^{*} \Psi}+\bar{\psi}}{\left|\pi^{*} \Psi+\phi\right|} \phi_{n}\right| \\
& \leq\left|1-\int_{\Delta_{n}} \frac{\overline{\phi_{n}}}{\mid \phi_{n}} \phi_{n}\right|+
\end{aligned}
$$

$$
\begin{gathered}
\left|\int_{\Delta_{n}}\left(\frac{\overline{\pi^{*} \Psi}+\bar{\psi}}{\left|\pi^{*} \Psi+\phi\right|}-\frac{\overline{\phi_{n}}}{\left|\overline{\phi_{n}}\right|}\right) \phi_{n}\right|+ \\
\left|\int_{\tilde{R} \backslash \Delta_{n}} \frac{\overline{\pi^{*} \Psi}+\bar{\psi}}{\left|\pi^{*} \Psi+\phi\right|} \phi_{n}\right| \\
\leq 1-\int_{\Delta_{n}}\left|\phi_{n}\right|+\frac{1}{2^{n}} \int_{\Delta_{n}}\left|\phi_{n}\right|+\int_{\tilde{R} \backslash \Delta_{n}}\left|\phi_{n}\right| \rightarrow 0 \\
\text { as } n \rightarrow \infty .
\end{gathered}
$$

On the other hand, there is a constant $C_{2}$ such that $\|\Phi\|_{1, V} \leq C_{2}\|\Phi\|_{1, R \backslash V}$ for any $\Phi \in$ $A_{2}^{1}(R)$. Hence

$$
\begin{aligned}
& \left|\int_{\tilde{R}} \frac{\overline{\pi^{*} \Psi}}{\left|\pi^{*} \Psi\right|} \phi_{n}\right|=\left|\int_{R} \frac{\bar{\Psi}}{|\Psi|} \Theta_{R \backslash \widetilde{R}} \phi_{n}\right| \leq\left(C_{2}+1\right) \\
& \quad \times \int_{R \backslash V}\left|\Theta_{R \backslash \tilde{R}} \phi_{n}\right| \leq\left(C_{2}+1\right) \int_{\tilde{R} \backslash \Delta_{n}}\left|\phi_{n}\right| \rightarrow 0,
\end{aligned}
$$

where $\Theta_{R \backslash \tilde{R}}: A_{2}^{1}(\tilde{R}) \rightarrow A_{2}^{1}(R)$ is the relative Poincaré series operator. This completes the proof.

\section{References}

[1] R. S. Hamilton: Extremal quasiconformal mappings with prescribed boundary values. Trans. Amer. Math. Soc., 138, 399-406 (1969).

[2] W. K. Hayman and E. Reich: On Teichmüller mappings of the disk. Complex Variables, 1, $1-12$ (1982).

[3] Huang Xinzhong: On the extremality for Teichmüller mappings (preprint).

[4] C. McMullen: Amenability, Poincaré series and quasiconformal maps. Invent. Math., 97, 95-127 (1989).

[5] H. Ohtake: On the norm of the Poincaré series operator for a universal covering group. J. Math. Kyoto Univ. , 32-1, 57-72 (1992).

[6] E. Reich and K. Strebel: Extremal quasiconformal mappings with given boundary values. Contributions to Analysis, a collections of Papers Dedicated to Lipman Bers (eds. L. V. Ahlfors et al.). Academic Press, New York, pp. 375-391 (1974).

[ 7 ] G. C. Sethares: The extremal property of certain Teichmüller mappings. Comment. Math. Helv., 43, 98-119 (1968).

[ 8 ] K. Strebel : Zur Frage der Eindeutigkeit extremaler quasikonformer Abbildungen des Einheitskreises. Comment. Math. Helv., 36, 306-323 (1962).

[9] K. Strebel: On lifts of extremal quasiconformal mappings. J. d'Analyse Math., 31, 191-203 (1977).

[10] K. Strebel: On quasiconformal mappings of open Riemann surfaces. Comment. Math. Helv., 53, 301-321 (1978) 